themselves (and in turn the public) thoughtfully and fully so as to be able to give cogent advice on behalf of that public ${ }^{1}$. (ii) The national committee should deal with ethical issues, including the infectious risk to the public ${ }^{1}$. This is critically different from the role of the FDA, which is charged with evaluating the safety and efficacy of drugs. In the case of xenotransplantation, there is a unique risk to third parties that raises special ethical issues that the FDA is not constituted to address.

Their third point, that one must proceed to clinical trials to understand the risk, misses our most important point: a thoughtful and appropriate deliberative process, demanding a high degree of public engagement, must precede the clinical trials that will reveal the risk. There are many experimental clinical procedures for which risk is uncertain. Yet, before those risks are imposed, consent must be obtained from individuals undergoing those risks. What is obligatory here, analogously, is that we obtain full discussion and assessment from a representative group before we put the public at risk. We believe that physician-scientists, medical centers, federal agencies such as the FDA, and commercial concerns should not impose risk on the public before such a process has been accomplished.

Lastly, we do not "wish to ignore" any aspect of the important work that has taken place to date, including that of the FDA. However, it is through a combination of many processes, including an appropriate discussion of the ethics, that we hope clinical xenotransplantation will be made acceptable and be allowed to proceed. Xenotransplantation research should be encouraged and pursued vigorously. Our call for a moratorium on clinical work is not anti-science; rather it is a way of respecting the rights of the public, thus preserving the trust of the public in science.

FriTz H. BACH, LACHLAN FORROW

Beth Israel Deaconess Medical Center

Harvard Medical School

Boston, MA 02215

\section{NORMAN DANIELS}

Tufts University

Boston, MA 02111

\section{HARVEY V. FINEBERG \\ Harvard University}

Cambridge, MA 02138

1. Bach, F.H. et al. Nature Med. 4, 141-144 (1998).

2. Fishman, J.A. Kidney Int!, 51, \$41-\$45 (1997).

\title{
Poor reporting of oocyte apoptosis
}

To the editor-We read with interest the report by Perez et al. on chemotherapymediated female germ cell destruction, the cover story for the November 1997 issue $^{1}$. The Sunday Times newspaper (London, UK) reported this research under the headline "Implant offers women lifelong fertility" and went on to suggest that both fumonisin- $\mathrm{B}_{1}$, a fungal toxin, and sphingosine-1phosphate could be used to stop cells in the ovaries from dying ${ }^{2}$. Although this is a far cry from Perez et al. reported, we were concerned to note that this erroneous interpretation was faithfully propagated around the globe, together with the implied sensationalism "implant of mysterious fungal toxin raises the possibility of 80 year old mothers".

Fumonisin- $\mathrm{B}_{1}\left(\mathrm{FB}_{1}\right)$ has been found to have specific, potent activity as an inhibitor of sphingolipid biosynthesis by blocking the conversion of sphinganine to ceramide ${ }^{3}$. Ceramide is a recently identified lipid second messenger that is believed to be one of the immediate signals for cell death generated in tumor cells treated with the chemotherapeutic agent, daunorubicin ${ }^{4}$. The production of ceramide and the ensuing onset of apoptosis in murine leukemia cells cultured in the presence of daunorubicin is prevented by pretreatment with $\mathrm{FB}_{1}$ (ref. 5). Perez et al. however showed that in haploid mouse ooctyes, the lethality of doxorubicin is blocked by pretreatment with sphingosine-1-phosphate, an endogenous downstream inhibitor of ceramide-promoted intracellular signaling, but not by $\mathrm{FB}_{1}$ (ref. 1).

The fumonisin mycotoxins are natural contaminants of corn infected with the fungus Fusarium moniliforme ${ }^{6}$. Ingestion of $\mathrm{FB}_{1}$, the major fumonisin produced by the fungus, causes a variety of toxicoses in animals, including equine leukoencephalomalacia and porcine pulmonary edema. Human consumption of Fusarium-contaminated corn products has been epidemiologically linked to increased rates of esophageal cancer in regions of the world in which corn is the staple grain, such as the Transkei region of South Africa and the Linxian province of China.

In rats, feeding with $\mathrm{FB}_{1}$ causes severe hepatotoxicity, cirrhosis and liver tumors ${ }^{7}$. Paradoxically, $\mathrm{FB}_{1}$ treatment causes marked apoptosis of hepatocytes in rats. This might be related to over expression of transforming growth factor beta 1 (TGF- $\beta 1$ ) by "activated" hepatic stellate (Ito) cells during $\mathrm{FB}_{1}$ feeding (manuscript in preparation). Cells undergoing apoptosis in turn secrete more TGF- $\beta 1$, thus setting up a positive feedback loop with stellate cell activation and more apoptosis. It is not clear whether the tumor promoting effects of $\mathrm{FB}_{1}$ are related to the disruption of sphingolipid metabolism that is caused by these mycotoxins.

The molecular mechanisms of action of $\mathrm{FB}_{1}$ are thus complex, and it appears that this fungal toxin may act as either an inhibitor or promoter of apoptosis, depending on the experimental situation and it could therefore be extremely risky to administer these potentially carcinogenic mycotoxins to humans. It is clear that the study by Perez et al. has caused intense world-wide public interest. However the reports in the lay press concerning $\mathrm{FB}_{1}$ and oocyte apoptosis were incorrect and misleading, and should be rebuked.

ERIC R. LEMMER

MRC/UCT Liver Research Centre

Groote Schuur Hospital

Observatory, Cape 7925, South Africa

email: eric@liver.uct.ac.za

Pauline De La M. Hall

Department of Anatomical Pathology

Flinders Medical Centre

Bedford Park, South Australia, 5042

Wentzel C.A. Gelderblom

$\&$ Walter F.O. Marasas

Programme on Mycotoxins and Experimental

Carcinogenesis (PROMEC)

Medical Research Council, PO Box 19070

Tygerberg 7505, South Africa

1. Perez, G.I., Knudson, C.M., Leykin, L., Korseyer, S.J. \& Tilly, J.L. Apoptosis-associated signaling pathways are required for chemotherapy-mediated female germ cell destruction. Nature Med. 3, 1228-1232 (1997).

2. Rogers, L. New implants will give women lifelong fertility. London Sunday Times, Nov. 9. p. 3, Sect. 1 (1997).

3. Wang, E., Norred, W.P., Bacon, C.W., Riley, R.T. \& Merril, A.H. Inhibition of sphingolipid biosynthesis by fumonisins. J. Biol. Chem. 266, 14486-14490 (1991).

4. Spiegel, S., Foster, D. \& Kolesnick, R.N. Signal transduction through lipid second messengers. Curr. Opin. Cell Biol. 8, 159-167 (1996).

5. Bose, R. et al. Ceramide synthase mediates daunorubicin-induced apoptosis: An alternative mechanism for generating death signals. Cell 82, 405-414 (1995).

6. Gelderblom, W.C.A. et al. Fumonisins: Novel mycotoxins with cancer promoting activity produced by Fusarium moniliforme. Appl. Environ. Microbiol. 54, 1806-1811 (1988)

7. Gelderblom, W.C.A., Kriek, N.P.J., Marasas, W.F.O. \& Thiel, P.G. Toxicity and carcinogenicity of the Fusarium moniliforme metabolite, fumonisin $B_{11}$ in rats. Carcinogenesis 12, 1247-1251 (1991). 